\title{
Critical care considerations in the post-operative period for the lung transplant patient
}

\author{
Josna Padiyar \\ Norton Thoracic Institute, St Joseph's Hospital and Medical Center, Phoenix, AZ, USA \\ Correspondence to: Josna Padiyar, DO. Norton Thoracic Institute, St Joseph's Hospital and Medical Center, 500 W Thomas Road, Suite 500, Phoenix, \\ AZ 85013, USA. Email: josna.padiyar@commonspirit.org.
}

\begin{abstract}
The post-operative management of a lung transplant recipient can be complex. Several factors including medical comorbidities, severity of illness at the time of transplant and intra-operative events can affect graft function and overall survival. During the immediate post-operative period, it becomes essential for early recognition of disease-specific sequelae as they can impact the patient's outcome and quality of life. This often necessitates a multidisciplinary team of pulmonologists, surgeons, medical sub-specialists as well as skilled nurses and respiratory therapists familiar with caring for these patients. Based on the experiences of a high-volume transplant center, this chapter will outline key considerations within each organ system in this specific patient population in the Intensive Care Unit.
\end{abstract}

Keywords: Lung transplantation; critical care; immediate post-operative period; multidisciplinary management

Submitted Sep 07, 2021. Accepted for publication Sep 17, 2021.

doi: $10.21037 /$ jtd-21-1441

View this article at: https://dx.doi.org/10.21037/jtd-21-1441

\section{Introduction}

The immediate post-operative management of a lung transplant recipient is critical to the short and longterm outcome of the patient. It begins with close communication between the anesthesiologist, surgeon and pulmonary intensivist. With a clear understanding of intra-operative hemodynamics, bleeding complications, echocardiographic findings, primary lung implantation and potential need for cardiopulmonary bypass, the receiving provider can develop an appropriate direction of care in the ICU.

Over the last 10 years in the United States, the average age of the lung transplant recipient has increased significantly. The percentage of patients over the age of 65 has quadrupled, from $6.9 \%$ in 2004 to almost $30 \%$ in 2016 (1). Optimizing comorbidities and preserving end organ function requires a multi-disciplinary, systems based approach. Therefore, based on the experiences of a large volume lung transplant center, we will use this method to describe management strategies and potential complications that can arise in this complex patient population.

\section{Neurologic considerations}

Upon arrival to the ICU, a complete neurologic assessment should be performed to establish a baseline of peripheral circulation and perfusion. Patients who experienced severe hemodynamic fluctuations intra-operatively are at higher risk for cerebrovascular ischemia or hemorrhage. Once a baseline neurologic status is established, patients should be maintained at light sedation with daily spontaneous awakening trials until extubation.

One of the most common post-operative neurologic complications after lung transplantation is delirium. Typically seen on ICU day 4, delirium carries with it an increased hospital and ICU LOS, time on mechanical ventilation and both in-hospital as well as one-year mortality (2). While there are several mood altering agents that can worsen delirium to all-comers in the ICU (benzodiazepines, opiates), this particular patient population is exposed to high dose steroids which places them at a higher risk for the development of delirium. Strategies for the management of steroid induced delirium are center specific. At our institution, if other disease processes are 
ruled out and conservative strategies are attempted without success, we will perform a faster taper of steroids with consideration to the lung allograft.

Calcineurin inhibitors (CNIs) have a rare association with posterior reversible encephalopathy syndrome (PRES) even in the absence of elevated blood pressure. There should be a high suspicion for PRES when other laboratory or radiographic findings are inconclusive. MRI is required for the diagnosis. While all CNIs can cause PRES, the management strategy is typically to change to a different agent within the same drug class. This will likely lead to resolution of encephalopathy.

Hyperammonemia syndrome (HS) is an extremely rare but important condition to discuss in this group of patients as it carries a mortality as high as $70 \%$ in some studies (3). The etiology is unclear, however there is an association with Ureaplasma spp. and Mycoplasma hominis isolates from airway specimens (3). It is proposed that there is an overgrowth of these urea-splitting organisms leading to the overproduction of ammonia. HS is characterized by an elevated serum ammonia level along with encephalopathy, abnormal liver function and seizures. The diagnosis is made based on clinical findings and serum ammonia levels greater than twice the upper limit of normal. Treatment strategies are center specific. At our institution, we will use antimicrobial therapy along with lactulose to reduce the production of ammonia from colonic bacteria. In addition, we will adjust the patient's diet to decrease protein intake.

\section{Cardiovascular/hemodynamic considerations}

Assessment of hemodynamics is a mainstay of immediate post operative management. It is important to have several tools to trend hemodynamics from the operating room to the ICU. Most patients will return from the OR with a pulmonary artery catheter and arterial line. These invasive lines can be used to estimate cardiac output/index, stroke volume, systemic vascular resistance, pulmonary artery pressure and CVP. These measurements alongside other markers of cardiac function (urine output, serum lactate) will dictate which vasoactive or inotropic agents to use post-op.

Intra-operative TEE findings can also play a role in the direction of management. It is important to make note of right and left ventricular size and function at the end of the case. Discussion points between the anesthesiologist and receiving intensivist include pulmonary artery pressures during chest closure, need for inhaled vasodilators or inotropic support, atrial arrhythmias, need for cardiopulmonary bypass, chest tube and urine output, blood loss and fluid resuscitation.

A baseline ECG on arrival to the ICU is helpful in several ways. First, it allows the receiving provider to make note of a baseline rhythm along. More importantly, it is critical to continuously evaluate for ST elevations which are consistent with air embolization down the coronary arteries. After implantation of the lung grafts, the pulmonary arteries are de-aired. It is possible for air to track down the coronaries. Specifically this can occur in the right coronary artery (RCA) due to its anterior location. The patient will present to the ICU with ECG findings of ST elevations in an RCA distribution. Clinically, they exhibit hypotension with a narrow pulse pressure, low cardiac index and rising lactate. The use of inotropes may help support these patients. Ultimately, if they cannot be supported with inotropic and vasoactive drugs, veno-arterial extracorporeal membrane oxygenation (VA ECMO) should be considered.

Based on several factors made by the operating surgeon, patients may return from the OR with an open chest. Bedside point of care ultrasound can be challenging in these patients so volume status determination should be made using other strategies such as pulse pressure variation, CVP and urine output. In order to avoid pulmonary edema, these patients will receive minimal intravenous fluid boluses. However, patients with chronic pulmonary infections (ex. cystic fibrosis) or those who require cardiopulmonary bypass (ex. pulmonary hypertension) can be profoundly hypotensive due to third spacing of fluid or vasoplegia, respectively. The use of targeted vasopressors with judicious intravenous fluid administration is key.

At our institution, the use of thoracic epidurals for adjunctive pain control has shown to be beneficial with minimizing intravenous or enteral narcotics. However, these patients can experience profound orthostatic hypotension. Reflexive intravenous fluid administration should be avoided. Treatment options include weaning the epidural to the patient's pain tolerance and educating the care team about slow movements from a seated to standing position.

The incidence of atrial arrhythmias is highest in the first 30 days after lung transplant (4). One study proposed this to be due to inflammation around bronchial anastomoses creating anatomic deformity in the native pulmonary vein antrum. Several centers use standardized treatment 
protocols for post operative arrhythmia as it can occur in up to $25 \%$ of lung transplant recipients (4). While atrial arrhythmias are a common occurrence in this patient population, it remains prudent to determine the etiology prior to management. If the rhythm is stable, reversible causes must be rule out or managed prior to treatment.

\section{Pulmonary considerations}

One of the first things to be done on arrival to the ICU is evaluate gas exchange which will help determine graft function. Our protocol is to obtain and arterial blood gas approximately 30 minutes after arrival to the ICU on 1.0 FiO2. We do not have a standardized PEEP setting as this can vary based on patients needs intra-op. If the patient required intraoperative inhaled vasodilator support such as inhaled epoprostenol or inhaled nitric oxide, it is resumed in the ICU for the initial blood gas. A chest radiograph is also performed at this time which helps determine the PGD0 score (5). It can also be used to ensure appropriate position of the endotracheal tube, pulmonary artery (PA) catheter, central venous lines, and chest tubes. Radiographs will allow evaluation of pleural spaces for effusions which at this stage, would likely be secondary to pleural bleeding.

Ventilator management includes low tidal volume ventilation with lung protective strategies. The goal is to target low peak inspiratory and driving pressures to avoid ventilator induced lung injury. Positive end expiratory pressure (PEEP) and $\mathrm{FiO} 2$ are adjusted in increments to maintain an $\mathrm{SpO} 2>90 \%$ and $\mathrm{PaO} 2>60 \mathrm{mmHg}$.

If the patient is stable from a respiratory standpoint, a diagnostic bronchoscopy with bronchial wash will be performed within the first 24 hours in the ICU. Following the initial evaluation, there is utility to routine use of bronchoscopy for therapeutic aspiration of secretions. We will typically perform a diagnostic bronchoscopy to optimize our patients prior to extubation. If there are significant secretions or sloughing, this may delay extubation and ultimately lead to early tracheostomy. During each bronchoscopy, the anastomoses are carefully inspected and graded (6).

Once extubated, patients may have difficulty coughing up secretions due to pain or diaphragmatic weakness. The use of mucolytics and chest physiotherapy using intrapulmonary devices can assist with mucous clearance.

\section{Pulmonary complications}

\section{Primary graft dysfunction (PGD)}

PGD is the most common cause of post-operative hypoxemia within the first 72 hours after transplantation (5). It is caused by ischemia reperfusion injury resulting in noncardiogenic pulmonary edema. The primary management is supportive care while maintaining euvolemia and avoiding ventilator-induced lung injury. Those patients who are refractory to optimized PEEP and inhaled vasodilators may require veno-venous extracorporeal support until PGD resolves.

\section{Airway complications}

Allograft airways depend on retrograde flow from the pulmonary arterial system due to the loss of bronchial circulation in the donor lung. Revascularization will occur by the recipient's bronchial circulation over 2 to 4 weeks, however in that time, the airways are at higher risk of ischemia and necrosis. This is due to the poorly oxygenated blood supply they receive from the pulmonary arterial system (7). In addition, longer ischemic times and infection can impact anastomotic healing. The International Society for Heart and Lung Transplantation (ISHLT) created a working group to define these airway complications (6). Every bronchoscopy performed after transplant should evaluate the anastomoses and distal airways for ischemia or dehiscence. If airway complications are suspected, chest computed tomography (CT) will aid in the evaluation of even small extraluminal abnormalities.

\section{Pleural complications}

Pleural effusions are common in the post-operative period. Due to long term sequelae of chronic effusions, an aggressive strategy is used to adequately drain the pleural space. In the immediate post-op setting, hemothorax is the concern. Recipients who develop large effusions despite functioning chest tubes and correction of coagulopathy may have to return to the operating room for exploration.

Chylothorax is a rare complication that results in accumulation of lymphatic fluid in the pleural space. Index of suspicion is increased with a large volume chest tube output that has a milky appearance. This can occur with 
surgical disruption of the thoracic duct or its branches. Triglyceride levels of the pleural fluid will be $>100$ $\mathrm{mg} / \mathrm{dL}$. Initial management is conservative using a parenteral non-fat nutrition regimen and intravenous octreotide. If these strategies are ineffective, thoracic duct ligation can be considered.

\section{Diaphragmatic dysfunction}

Diaphragmatic dysfunction or paralysis can occur due to injury to the phrenic nerve. A chest radiograph with an elevated hemidiaphragm in the absence of positive pressure ventilation in pathognomonic. If symptomatic, surgical plication can be considered.

\section{Stenosis of the pulmonary artery anastomosis}

Stenosis of the pulmonary artery anastomosis presents as persistent pulmonary hypertension after transplantation. This diagnosis is considered in patients who maintain high pulmonary artery pressures despite adequate blood pressure control and inhaled vasodilator therapy. Early discussion with the Cardiothoracic Surgery team can elucidate the likelihood of this diagnosis.

\section{Thrombosis or stenosis of the pulmonary veins}

Thrombosis or stenosis of the pulmonary veins presents as persistent alveolar infiltrates of the effected side on chest radiograph. This diagnosis can be challenging as it mimics other early causes of hypoxemia including reperfusion edema or infection. Clinical findings include recurrent pleural effusions, impaired gas exchange and hemodynamic instability. Index of suspicion should increase in those patients who are suspected to have primary graft dysfunction but do not improve with supportive care. The diagnosis is made on transesophageal echocardiography evaluating pulmonary vein velocities.

\section{Pulmonary embolism (PE)}

Acute PE has a deleterious effect on this patient population due to the lack of bronchial artery circulation in the early post-operative period. If impaired gas exchange occurs in the absence of radiographic findings, acute $\mathrm{PE}$ can be considered. Diagnosis is made with CT angiography. In those patients who will tolerate it, full dose anticoagulation should be started.

\section{Vocal cord paralysis}

Vocal cord paralysis can occur due to recurrent laryngeal nerve injury following lung transplantation. This places patients at a higher risk of aspiration which can contribute to chronic lung allograft dysfunction (CLAD). If there is abnormal movement of the vocal cords, avoiding oropharyngeal nutrition and early consultation of an Ear Nose and Throat (ENT) specialist is beneficial.

\section{Immunosuppression}

An appropriate immunosuppressive regimen is necessary to preserve long term allograft function. While protocols may differ slightly based on each center's practice pattern, the general principals remain the same. Commonly used immunosuppressive pharmacotherapy can be divided into induction and maintenance agents.

Induction therapy includes agents that decrease the $\mathrm{T}$ cell immune response to the implanted graft such as thymoglobulin (antithymocyte globulin) or antiinterleukin-2 receptor antagonist monoclonal antibodies (basiliximab or daclizumab). Highly sensitized patients may require the use of rituximab or anti-CD-52 monoclonal antibodies. At our center, high dose steroids (methylprednisolone $10 \mathrm{mg} / \mathrm{kg}$ ) are also administered in the peri-operative period.

Maintenance therapy includes steroids, pyrimidine synthesis inhibitors (mycophenolate or azathioprine) and calcineurin inhibitors (tacrolimus or cyclosporin). Triple therapy is typically started within the first 24 hours after implantation and continued for the remainder of the transplant recipient's lifetime.

\section{Infectious disease considerations}

Lung transplant recipients are especially susceptible to pulmonary infections due to constant exposure to external stimuli and impaired ciliary clearance. In the first few months, the predominant infections are bacterial followed by viral and fungal.

At our institution, antimicrobial prophylaxis includes broad spectrum gram-positive, gram-negative and 
atypical coverage with Vancomycin, Cefepime and a fluoroquinolone. Diagnostic bronchoscopy with bronchial wash $(\mathrm{BW})$ is performed within the first 24 hours of implantation. Antibiotics are de-escalated based on donor and recipient cultures after 72 hours. Cytomegalovirus (CMV) prophylaxis is initiated with oral valganciclovir. Pneumocystis Jirovecii prophylaxis is started with trimethoprim/sulfamethoxazole. Routine fungal prophylaxis at our center includes inhaled amphotericin along with oral itraconazole.

Nosocomial infections are common in lung transplant recipients in the early postoperative period. While invasive fungal and CMV infections are rare during this stage, bacterial pneumonia due to pseudomonas aeruginosa or staphylococcus aureus predominate (8).

Other infections related to the immediate post-operative period include mediastinitis after sternotomy, CMV viremia in the mismatched recipient, tracheobronchitis aspergillosis (TBA) occurring at the site of anastomosis and candidemia secondary to vascular catheters, to name a few. With the amount of immunosuppression required to maintain allograft function, there is a higher morbidity associated with these infections. Transplant infectious disease specialists are an invaluable resource as they can provide guidance for targeted antimicrobial therapy and adjust medications based on side effect profiles.

\section{Renal considerations}

Renal injury is common in the lung transplant recipient. Some studies report that close to $60 \%$ of patients experience some form of AKI in the early post-operative setting (9). Potential risk factors include underlying chronic kidney disease, peri-operative hemodynamic instability, prolonged mechanical ventilation, advanced age and use of calcineurin inhibitors (CNIs).

The mechanism of CNI induced renal injury is direct vasoconstriction of the afferent and efferent arterioles leading to decreased renal blood flow. These agents can cause severe electrolyte disturbances including hyperkalemia, hyponatremia and hypomagnesemia. When renal injury occurs, plasma CNI levels can rise and further worsen the renal dysfunction; therefore levels should be monitored with dose adjustments as needed.

Management strategies in these patients are supportive. The most important goal is to avoid prolonged blood pressure abnormalities, monitor urine output closely and maintain euvolemia. At our institution, we will consider early renal replacement therapy as a strategy to maintain an adequate volume status to decrease length of time on mechanical ventilation and avoid renal congestion.

\section{Gastrointestinal/hepatobiliary considerations}

Gastrointestinal complications are associated with substantial morbidity in the post-lung transplantation population $(10,11)$. Bilateral lung recipients are at higher risk and complications can include gastroparesis, gastroesophageal reflux, ileus and colorectal disease.

It is well demonstrated that pre-operative gastroesophageal reflux disease results in high risk for chronic rejection due to aspiration. With the addition of high dose steroids, mechanical ventilation and medications that delay gastric emptying (i.e., narcotics, anticholinergics), patients with a history of reflux are at especially high risk of acute aspiration events. Our institution's protocol includes a speech-language pathologist (SLP) consult along with a fiberoptic endoscopic evaluation of swallowing (FEES) prior to administering an oropharyngeal diet. If the patient shows signs of aspiration, gastrojejunal feeding tube can be considered.

Management strategies for ileus and colonic dilation include adjunctive non-opioid analgesia, early mobilization and electrolyte repletion. Preemptive involvement of colorectal consultants is beneficial as disease sequelae can have profound consequences in the outcome of these patients. A dreaded consequence of colonic dilation is perforation. Diagnostic criteria are similar to the nontransplant population (i.e., abdominal distention, air under diaphragm, elevated serum lactate). Due to high dose steroids and post operative opioids however, these patients are at higher risk. Management includes emergent surgical evaluation. These patients can have a profound morbidity if surgical repair results in end-ostomy.

C. difficile infection (CDI) has a mortality association in lung transplant recipients during the first 6 months of transplant (12). Common clinical criteria to diagnose CDI may be blunted in this patient population, so a high index of suspicion is important; especially if the patient is showing early signs of sepsis.

It is important to keep in mind the nutritional requirements of the post-transplant patient due to surgical wound healing, metabolic demands and graft survival (13). 
Several conditions are specific to the immediate post lung transplant patient which include primary graft dysfunction (minimize free water/sodium), post-surgical chylothorax, hyperammonemia syndrome, pancreatic insufficiency in cystic fibrosis and hypercalcemia in sarcoidosis. There is great utility to the nutritional consultant during daily ICU rounds in order to address disease specific conditions that require the adjustment of nutritional supplementation.

\section{Conclusion}

Caring for the post-operative lung transplant recipient requires a multidisciplinary approach. It begins with a detailed transition of care from the operating room to the ICU and requires attention to detail in the delivery of that care. The approach to managing these patients in the early post-operative period can play a major role in their long-term morbidity and mortality. Therefore, a group of primary intensivists, surgeons and subspecialists can have a synergistic effect in the management of these complex patients.

\section{Acknowledgments}

Funding: None.

\section{Footnote}

Provenance and Peer Review: This article was commissioned by the Guest Editor (Jonathan D'Cunha) for the series "Lung Transplantation: Past, Present, and Future" published in Fournal of Thoracic Disease. The article has undergone external peer review.

Conflicts of Interest: The author has completed the ICMJE uniform disclosure form (available at https:// dx.doi.org/10.21037/jtd-21-1441). The series "Lung Transplantation: Past, Present, and Future" was commissioned by the editorial office without any funding or sponsorship. The author has no other conflicts of interest to declare.

Ethical Statement: The author is accountable for all aspects of the work in ensuring that questions related to the accuracy or integrity of any part of the work are appropriately investigated and resolved.
Open Access Statement: This is an Open Access article distributed in accordance with the Creative Commons Attribution-NonCommercial-NoDerivs 4.0 International License (CC BY-NC-ND 4.0), which permits the noncommercial replication and distribution of the article with the strict proviso that no changes or edits are made and the original work is properly cited (including links to both the formal publication through the relevant DOI and the license). See: https://creativecommons.org/licenses/by-nc-nd/4.0/.

\section{References}

1. Courtwright A, Cantu E. Lung transplantation in elderly patients. J Thorac Dis 2017;9:3346-51.

2. Sher Y, Dhillon G, Maldonado J. Delirium After Lung Transplantation: Occurrence and Its Relationship to Morbidity and Mortality. J Heart Lung Transplant 2015;34:S173.

3. Buzo BF, Preiksaitis JK, Halloran K, et al. Association between Mycoplasma and Ureaplasma airway positivity, ammonia levels, and outcomes post-lung transplantation: A prospective surveillance study. Am J Transplant 2021;21:2123-31.

4. Chaikriangkrai K, Jyothula S, Jhun HY, et al. Incidence, Risk Factors, Prognosis, and Electrophysiological Mechanisms of Atrial Arrhythmias after Lung Transplantation. JACC Clin Electrophysiol 2015;1:296-305.

5. Snell GI, Yusen RD, Weill D, et al. Report of the ISHLT Working Group on Primary Lung Graft Dysfunction, part I: Definition and grading-A 2016 Consensus Group statement of the International Society for Heart and Lung Transplantation. J Heart Lung Transplant 2017;36:1097-103.

6. Crespo MM, McCarthy DP, Hopkins PM, et al. ISHLT Consensus Statement on adult and pediatric airway complications after lung transplantation: Definitions, grading system, and therapeutics. J Heart Lung Transplant 2018;37:548-563.

7. Fuller J, Fisher AJ. An update on lung transplantation. Breathe 2013;9:188-200.

8. Czebe K, Antus B, Varga M, et al. Pulmonary infections after lung transplantation. Orv Hetil 2008;149:99-109.

9. George TJ, Arnaoutakis GJ, Beaty CA, et al. Acute kidney injury increases mortality after lung transplantation. Ann Thorac Surg 2012;94:185-92.

10. Lahon B, Mordant P, Thabut G, et al. Early severe digestive complications after lung transplantation. Eur J 
Cardiothorac Surg 2011;40:1419-24.

11. Maurer JR. The spectrum of colonic complications in a lung transplant population. Ann Transplant 2000;5:54-7.

12. Lee JT, Kelly RF, Hertz MI, et al. Clostridium difficile infection increases mortality risk in lung transplant

Cite this article as: Padiyar J. Critical care considerations in the post-operative period for the lung transplant patient. J Thorac Dis 2021;13(11):6747-6753. doi: 10.21037/jtd-21-1441 recipients. J Heart Lung Transplant 2013;32:1020-6.

13. Jomphe V, Lands LC, Mailhot G. Nutritional Requirements of Lung Transplant Recipients: Challenges and Considerations. Nutrients 2018;10:790. 